\title{
NUMERICAL CALCULATION OF THE ELECTRONIC STRUCTURE FOR THREE-DIMENSIONAL QUANTUM DOTS
}

HEINRICH VOSS *

Institute of Mathematics, Hamburg University of Technology, D-21071 Hamburg, Germany

Abstract. In some recent papers Li, Voskoboynikov, Lee, Sze and Tretyak suggested an iterative scheme for computing the electronic states of quantum dots and quantum rings taking into account an electron effective mass which depends on the position and electron energy level. In this paper we prove that this method converges globally and linearly in an alternating way, i.e. yielding lower and upper bounds of a predetermined energy level in turn. Moreover, taking advantage of the Rayleigh functional of the governing nonlinear eigenproblem, we propose a variant which converges even quadratically thereby reducing the computational cost substantially. Two examples of finite element models of quantum dots of different shapes demonstrate the efficiency of the method.

Key words. quantum dot, electronic structure, electron states, computer simulation, nonlinear eigenproblem, Schrödinger equation, Rayleigh functional

PACS 73.20.At, 73.61.Ey

1. Introduction. Semiconductor nanostructures have attracted tremendous attention in the past few years because of their unique physical properties and their potential for applications in micro- and optoelectronic devices. In such nanostructures, the free carriers are confined to a small region of space by potential barriers, and if the size of this region is less than the electron wavelength, the electronic states become quantized at discrete energy levels. The ultimate limit of low dimensional structures is the quantum dot, in which the carriers are confined in all three directions. Therefore, a quantum dot can be thought of as an artificial atom.

In recent papers [1-3] Li, Voskoboynikov, Lee, Sze and Tretyak proposed an iterative scheme called Full Approximation Method (FAM) for computing the electron states of quantum dots assuming the effective one-band Hamiltonian approximation, the position and energy dependent quasi-particle effective mass approximation, the finite hard wall confinement potential, and the Ben Daniel-Duke boundary condition. The same method was applied in $[4,5]$ to study the effect of shape and size on electron transition energies, in $[6,7]$ where spin-orbit interaction was considered additionally, and in $[1,8-11]$ for simulating quantum rings.

The energy level $E$ and the wave function $\Phi$ satisfy the Schrödinger equation

$$
H\left(m_{j}\right) \Phi=E \Phi
$$

where the electron effective mass $m_{j}$ is a rational function $m_{j}=f_{j}(E)$ of the energy level for the quantum $\operatorname{dot}(\mathrm{j}=\mathrm{q})$ and the substrate surrounding the quantum dot called matrix $(\mathrm{j}=\mathrm{m})$. Mathematically, this is a nonlinear eigenvalue problem. Li et al. suggested to compute the $k$ :th energy level by the following full approximation method: Given an initial $E^{0}$, determine the corresponding effective mass $m_{j}^{0}=f_{j}\left(E^{0}\right), j \in\{q, m\}$, and for $n=1,2, \ldots$ iterate as follows: Compute the $k$ :th smallest eigenvalue $E^{n}$ of the linear problem

$$
H\left(m_{j}^{n-1}\right) \Phi^{n}=E^{n} \Phi^{n}, \quad \text { and update } m_{j}^{n}=f_{j}\left(E^{n}\right) .
$$

$\mathrm{Li}$ et al. report that in different situations this method converges, and in $[1-3,5,6]$ they even claim that the convergence is monotonical. A proof of convergence is not contained in the literature.

\footnotetext{
*E-mail address: voss@tu-harburg.de, Tel. +49 4042878 3279, FAX +49 40428782696
} 
In this paper we prove that the iterative process (1.2) converges globally for every eigenvalue of problem (1.1), if the electron effective mass is defined by the Kane formula [12-14] (cf. (2.2)). The iterates $E^{n}$ are alternately upper and lower bounds of its limit (contradicting the monotonical convergence stated by Li et al.), and the convergence is linear.

Taking advantage of the Rayleigh functional of the nonlinear eigenproblem (1.1) one gets a variant of FAM which is even quadratically convergent. The additional cost of this modification is negligible for the following reason. Discrete versions of (1.1) usually will be large and sparse, and in every iteration step of FAM one has to determine the $k$ :th smallest eigenvalue of a linear eigenproblem of this type. To evaluate the Rayleigh functional one has to solve a nonlinear real equation which depends on the corresponding eigenvector. This does not increase the computational cost considerably since sparse eigensolvers like the Lanczos method or Jacobi-Davidson method determine eigenvalues and eigenvectors simultaneously.

Our paper is organized as follows. Section 2 introduces the model under consideration and the numerical method of Li et al. In Section 3 we discuss the global and alternating convergence of the method, and the quadratic convergence of its variant. The proofs are left to Appendix A. In Section 4 we demonstrate the improvement by the modification of FAM for two three-dimensional small InAs/GaAs quantum dot heterostructures of different shapes. One is rotational symmetric with conical shape such that the Schrödinger equation can be reduced to a two-dimensional problem, and the other one is of pyramidal shape. The paper closes with concluding remarks.

2. The governing Schrödinger equation. We consider the problem to compute relevant energy states and corresponding wave functions of a three dimensional semiconductor quantum dot. Let $\Omega_{q} \subset \mathbb{R}^{3}$ be a domain occupied by the quantum dot, which is embedded in a bounded matrix $\Omega_{m}$ of different material. A typical example is an InAs quantum dot embedded in a GaAs matrix.

We consider the one-band envelope-function formalism for electrons and holes in which the effective Hamiltonian is given by

$$
\hat{H}=-\frac{\hbar^{2}}{2} \nabla \cdot\left(\frac{1}{m(E, x)} \nabla\right)+V(x)
$$

where $\hbar$ is the reduced Planck constant, and $\nabla$ denotes the spatial gradient. The electron effective mass $m(E, x)$ is assumed to be constant on the quantum dot and the matrix for every fixed energy level $E$, and is taken as [12-14]

$\frac{1}{m_{j}(E)}:=\left.\frac{1}{m(E, x)}\right|_{x \in \Omega_{j}}=\frac{P_{j}^{2}}{\hbar^{2}}\left(\frac{2}{E+E_{g, j}-V_{j}}+\frac{1}{E+E_{g, j}-V_{j}+\Delta_{j}}\right), \quad j \in\{m, q\}$

where the confinement potential $V_{j}:=\left.V\right|_{\Omega_{j}}$ is piecewise constant, and $P_{j}, E_{g, j}$ and $\Delta_{j}$ are the momentum matrix element, the band gap, and the spin-orbit splitting in the valence band gap for the quantum $\operatorname{dot}(j=q)$ and the matrix $(j=m)$, respectively.

To determine the relevant energy states and corresponding wave functions we have to solve the governing Schrödinger equation

$$
-\nabla \cdot\left(\frac{\hbar^{2}}{2 m_{j}(E)} \nabla \Phi\right)+V(x) \Phi=E \Phi, x \in \Omega_{q} \cup \Omega_{m} .
$$

Since the wave function decays outside the quantum dot very rapidly, it is reasonable to assume homogeneous Dirichlet conditions $\Phi=0$ on the outer boundary of $\Omega_{m}$, 
and on the interface between the quantum dot and the matrix the Ben Daniel-Duke condition [15] holds

$$
\left.\frac{1}{m_{q}} \frac{\partial \Phi}{\partial n_{q}}\right|_{\partial \Omega_{q}}=\left.\frac{1}{m_{m}} \frac{\partial \Phi}{\partial n_{m}}\right|_{\partial \Omega_{m}}, x \in \partial \Omega_{q} \cap \partial \Omega_{m}
$$

Here $n_{q}$ and $n_{m}$ denote the outward unit normal on the boundary of $\Omega_{q}$ and $\Omega_{m}$, respectively.

To determine the $k$ :th energy level $E_{k}$ and the corresponding wave function $\Phi_{k}$ of the quantum dot $\mathrm{Li}$ et al. suggested the following algorithm which they called Full Approximation Method in [7]:

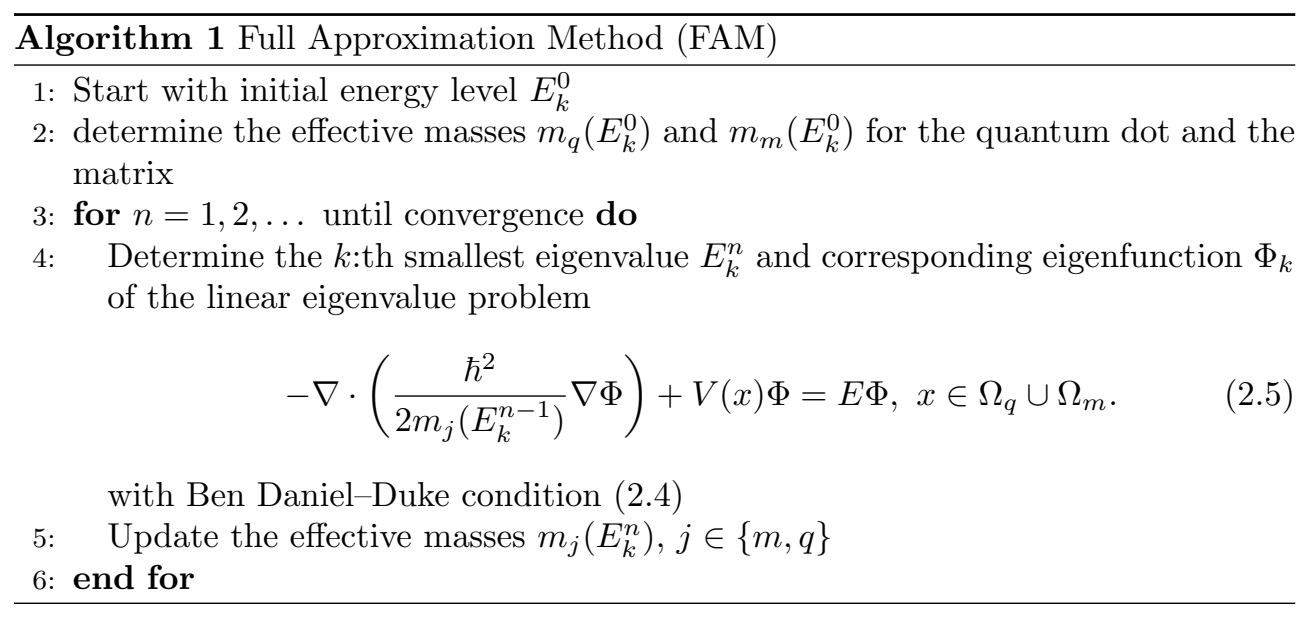

3. Convergence results. To analyze the iterative method of the last section we consider the linear eigenproblem

$$
-\nabla \cdot\left(\frac{\hbar^{2}}{2 m_{j}(\mu)} \nabla \Phi\right)+V(x) \Phi=E \Phi, x \in \Omega_{q} \cup \Omega_{m} .
$$

with interface condition (2.4) and homogeneous Dirichlet condition on the outer boundary of $\Omega_{m}$, which depends on a nonnegative parameter $\mu$.

Multiplying (3.1) by $\Psi$ in the Sobolev space $H_{0}^{1}(\Omega), \Omega:=\bar{\Omega}_{q} \cup \Omega_{m}$, and integrating by parts one gets the variational form of the Schrödinger equation

$$
\begin{gathered}
a(\Phi, \Psi ; \mu):=\frac{\hbar^{2}}{2 m_{q}(\mu)} \int_{\Omega_{q}} \nabla \Phi \cdot \nabla \Psi d x+\frac{\hbar^{2}}{2 m_{m}(\mu)} \int_{\Omega_{m}} \nabla \Phi \cdot \nabla \Psi d x+V_{q} \int_{\Omega_{q}} \Phi \Psi d x \\
\quad+V_{m} \int_{\Omega_{m}} \Phi \Psi d x=\lambda \int_{\Omega} \Phi \Psi d x=: \lambda b(\Phi, \Psi) \quad \text { for every } \Psi \in H_{0}^{1}(\Omega) .
\end{gathered}
$$

For $E_{g, j}-V_{j}>0, j \in\{m, q\}$ and every $\mu \geq 0$ the bilinear form $a(\cdot, \cdot ; \mu)$ is symmetric, bounded, and $H_{0}^{1}(\Omega)$-elliptic, and $b(\cdot, \cdot)$ is bilinear, positive definite, bounded, and completely continuous on $H_{0}^{1}(\Omega)$. Hence, the linear eigenproblem (3.1) has a countable set of eigenvalues $0<\lambda_{1}(\mu) \leq \lambda_{2}(\mu) \leq \cdots \rightarrow \infty$, each of finite multiplicity. Obviously, $E_{k}$ is the $k$ :th smallest eigenvalue of the nonlinear Schrödinger equation (2.3) if and only if $E_{k}$ is a fixed point of the real mapping $\mu \mapsto \lambda_{k}(\mu)$. 
The Rayleigh quotient

$$
R(\Phi ; \mu):=\frac{a(\Phi, \Phi ; \mu)}{b(\Phi, \Phi)}
$$

is monotonically decreasing with respect to $\mu \geq 0$ for every fixed $\Phi \in H_{0}^{1}(\Omega), \Phi \neq 0$. Hence, it follows from the minmax characterization of the eigenvalues of the linear eigenvalue problem (3.1) that for every fixed $k$ the mapping $\mu \mapsto \lambda_{k}(\mu)$ is monotonically decreasing with respect to $\mu$ from which the alternating behaviour of the iterates of the FAM follows. Theorem 3.1 describes the convergence of the FAM. Its proof is given in Appendix A.

TheOrem 3.1. Assume that $c_{j}:=E_{g, j}-V_{j}>0$ for $j \in\{m, q\}$, and let $E_{k}$ and $\lambda_{k}(\mu)$ be the $k$ :th smallest eigenvalue of the nonlinear Schrödinger equation (2.3) and the parameter dependent equation (3.1), both with Ben Daniel-Duke condition (2.4) on the interface $\Omega_{q} \cap \Omega_{m}$ and homogeneous boundary condition on the outer boundary of $\Omega_{m}$.

Let $E^{0} \geq 0$ be any initial value, and set $E^{n}:=\lambda_{k}\left(E^{n-1}\right)$ for $n \in \mathbb{N}$. Then it holds

$$
E^{0}<E^{2} \leq \cdots \leq E^{2 n-2} \leq E^{2 n} \leq E_{k} \leq E^{2 n+1} \leq E^{2 n-1} \leq \ldots E^{3} \leq E^{1},
$$

$$
\lim _{n \rightarrow \infty} E^{n}=E_{k},
$$

and the convergence is linear, i.e. there exists a constant $C, 0<C<1$ and $N \in \mathbb{N}$ such that

$$
\left|E^{n}-E_{k}\right| \leq C\left|E^{n-1}-E_{k}\right| \quad \text { for every } n \in \mathbb{N}, n \geq N
$$

The convergence behavior of FAM can be improved considerably if we take advantage of the Rayleigh functional. The variational form of the Schrödinger equation (2.3) is given by

Find $E \geq 0$ and $\Phi \in H_{0}^{1}(\Omega), \Phi \neq 0$ such that

$$
a(\Phi, \Psi, E)=E b(\Phi, \Psi) \quad \text { for every } \Psi \in H_{0}^{1}(\Omega) .
$$

For every fixed $\Phi \in H_{0}^{1}(\Omega), \Phi \neq 0$ the function $\lambda \mapsto a(\Phi, \Phi, \lambda)$ is monotonically decreasing and positive, and therefore the real equation

$$
f(\lambda ; \Phi):=\lambda b(\Phi, \Phi)-a(\Phi, \Phi, \lambda)=0
$$

has a unique positive solution $\lambda=: p(\Phi)$. Hence, equation (3.8) defines a real functional $p$ on $H_{0}^{1}(\Omega) \backslash\{0\}$ which we call the Rayleigh functional of the nonlinear eigenvalue problem (3.7).

If the quadratic form $a$ does not dependent on $\lambda$, then the Rayleigh functional $p$ is just the well known Rayleigh quotient. It is remarkable, that $p$ has similar properties as the Rayleigh quotient (cf. $[16,17]$ ): The eigenvalues $E_{1} \leq E_{2} \leq \ldots$ of problem (3.7) can be characterized as minmax values of the Rayleigh functional,

$$
E_{k}=\min _{\operatorname{dim} V=k} \max _{\Phi \in V, \Phi \neq 0} p(\Phi),
$$

and the corresponding eigenelements are stationary elements of $p$, i.e.

$$
p^{\prime}\left(\Phi_{k}\right)=0 \quad \text { for every eigenelement } \Phi_{k} \text { of (3.7). }
$$




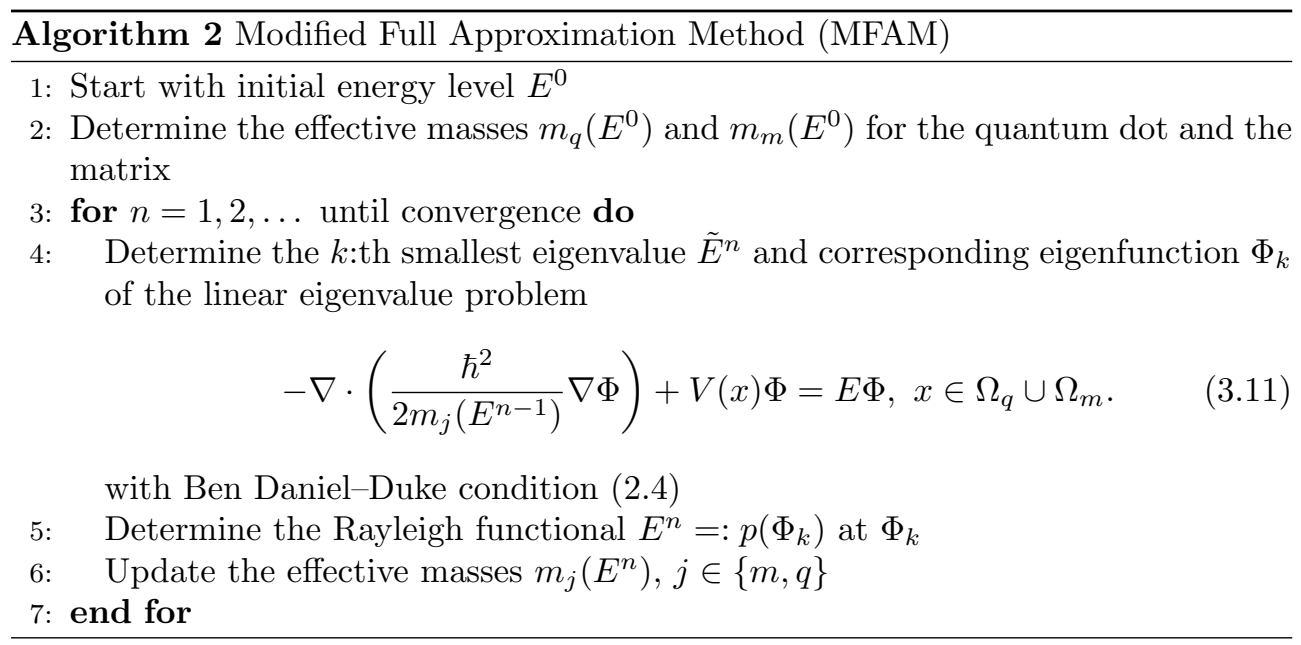

These properties suggest the modification of the full approximation method for computing the $k$ :th energy level of (2.3) in Algorithm 2.

The Modified Full Approximation Method (MFAM) is nothing else but the fixed point iteration $E^{n+1}=h\left(E^{n}\right):=p\left(\Phi_{k}\left(\tilde{E}^{n-1}\right)\right)$, and since $\Phi\left(E_{k}\right)$ is a stationary element of $p$ it follows that $h^{\prime}\left(E_{k}\right)=0$. Hence [18], the Modified Full Approximation Method converges quadratically to $E_{k}$, i.e. there exist some constant $C>0$ such that

$$
\left|E^{n}-E_{k}\right| \leq C\left|E^{n-1}-E_{k}\right|^{2} \quad \text { for every } n \in \mathbb{N} .
$$

We discussed the Full Approximation Method and its modification for the infinite dimensional Schrödinger equation (2.3). Discretizing it by a Galerkin method (finite elements, e.g.) one gets a rational matrix eigenvalue problem

$$
\lambda M x-\frac{1}{m_{q}(\lambda)} A_{q} x-\frac{1}{m_{m}(\lambda)} A_{m} x-B x=0
$$

where

$$
\begin{gathered}
A_{j}=\left(\int_{\Omega_{j}} \nabla \Phi_{k} \cdot \nabla \Phi_{\ell} d x\right)_{k, \ell}, j \in\{q, m\} \\
M=\left(\int_{\Omega} \Phi_{k} \Phi_{\ell} d x\right)_{k, \ell} \text { and } B=\left(V_{q} \int_{\Omega_{q}} \Phi_{k} \Phi_{\ell} d x+V_{m} \int_{\Omega_{m}} \Phi_{k} \Phi_{\ell} d x\right)_{k, \ell}
\end{gathered}
$$

and $\Phi_{k}$ denotes a basis of the ansatz space.

$A_{q}, A_{m}$ and $B$ are symmetric and positive semi-definite, and $M$ is positive definite, and for $\lambda \geq 0$ the matrix

$$
\frac{\hbar^{2}}{2 m_{q}(\lambda)} A_{q}+\frac{\hbar^{2}}{2 m_{q}(\lambda)} A_{q}
$$

is positive definite. Hence, all considerations for the infinite dimensional problem hold for the finite element discretization as well, and therefore the Full Approximation Method for (3.13) converges globally and linear, and its modification is quadratically convergent. Moreover, it follows from the minmax characterization (3.9) of the 
FIG. 4.1. Conical quantum dot: triangulation

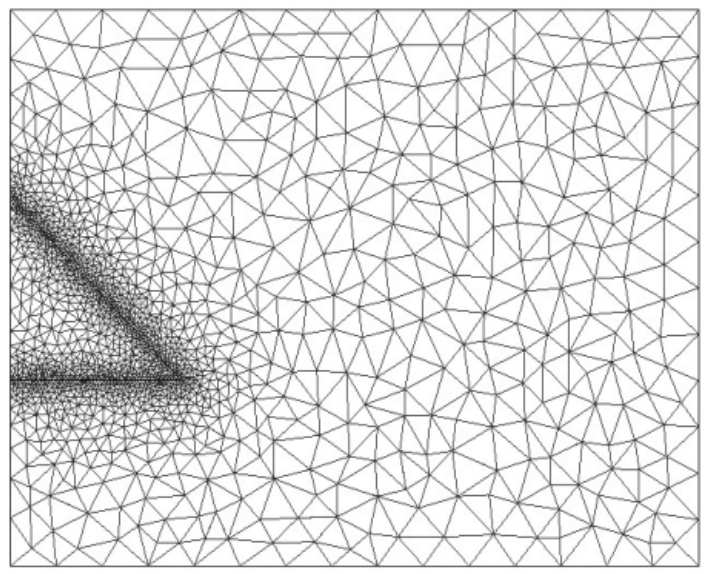

nonlinear Schrödinger equation that the $k$ :th smallest eigenvalues of the discretized problem (3.13) is an upper bound of the corresponding eigenvalue of problem (2.3).

Other discretization schemes like finite differences or finite volume methods also yield nonlinear matrix eigenvalue problems like (3.13). If the matrices share the symmetry and definiteness properties mentioned in the last paragraph, then FAM and MFAM have the convergence properties established above. Notice however, that the eigenvalues of the discretized problem are usually not upper bounds of the eigenvalues of the infinite dimensional problem. For instance this is the case for a finite volume scheme for the nonlinear Schrödinger equation (2.3) modeling a pyramidal quantum dot problem which was proposed by Hwang, Lin, Wang and Wang [19, 20].

4. Numerical results. In this section we demonstrate the convergence of the Full Approximation Method and its modification for two examples, a conical quantum dot, where we use its rotational symmetry to reduce the size of the discretized problem, and a pyramidal quantum dot.

4.1. Conical quantum dot. We consider a conical quantum dot with radius $R_{0}=10 \mathrm{~nm}$ and height $z_{0}=10 \mathrm{~nm}$, which is embedded in cylindrical matrix with Radius $R_{1}=40 \mathrm{~nm}$ and height $z_{1}=30 \mathrm{~nm}$. Since the system is rotationally symmetric, the wave function can be written (in cylindrical coordinates) as $\Phi(x)=\phi(r, z) \exp (i \ell \varphi)$, where $\ell=0, \pm 1, \pm 2, \ldots$ is the electron orbital quantum number. Then the Schrödinger equation obtains the form

$$
-\frac{\hbar^{2}}{2 m_{j}(E)}\left(\frac{1}{r} \frac{\partial}{\partial r}\left(r \frac{\partial}{\partial r}\right)+\frac{\partial^{2}}{\partial z^{2}}-\frac{\ell^{2}}{r^{2}}\right) \phi(r, z)+V_{j} \phi(r, z)=E \phi(r, z),
$$

with $(r, z) \in(0,40) \times(0,30)$, and the Ben Daniel-Duke interface condition. The boundary conditions are $\phi(r, 0)=\phi(r, 30)=0,0 \leq r \leq 40$, and $\phi(40, z)=0$, $0 \leq z \leq 30$, and for continuity reasons $\frac{\partial}{\partial r} \phi(0, z)=0$ for $\ell=0$ and $\phi(0, z)=0$ for $\ell \geq 1$.

Using FEMLAB [21] we discretized this problem by quadratic finite elements on a triangular net, which was refined close to the boundary of the conical quantum dot (cf. Figure 4.1). We obtained a rational matrix eigenvalue problem of dimension 6720 for $\ell=0$ and 6653 for $\ell \geq 1$, which satisfies the conditions of Section 3. Since the matrices 


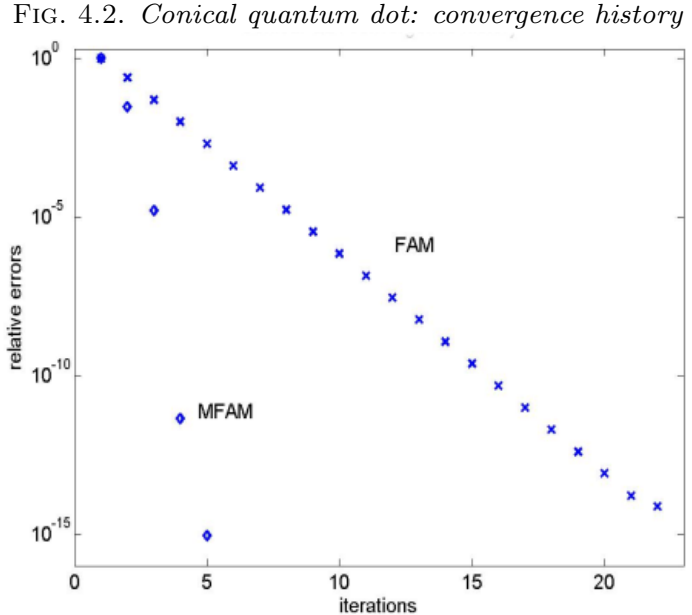

in (3.13) are sparse we solved the linear eigenproblems corresponding to (3.1) by the implicitly restarted Arnoldi method ARPACK [22] (which is called by the function eigs in MATLAB). The convergence history for the linearly convergent method and its quadratically convergent variant for the ground state (smallest eigenvalue for $\ell=0$ ) are contained in Figure 4.2. Notice that the additional cost of the MFAM upon FAM is negligible because sparse eigensolvers approximate eigenvalues and eigenvectors simultaneously.

We used the following semiconductor band structure parameters [23]: For the InAs quantum dot $P_{q}=0.8503, E_{g, q}=0.42, \Delta_{q}=0.48$ and $V_{q}=0$, and for the GaAs matrix $P_{m}=0.8878, E_{g, m}=1.52, \Delta_{m}=0.34$ and $V_{m}=0.7$. Table 4.1 contains the CPU time and the number of iterations to approximate the 12 smallest eigenvalues of (4.1) by FAM and MFAM. In all cases the initial approximation was $E^{0}=0$. The methods were stopped if the relative error was less than $10^{-6}$, which can be guaranteed by the alternating nature of the iterates of FAM. The numerical tests were performed on an Intel Centrino $\mathrm{M}$ processor with $1.7 \mathrm{GHz}$ and $1 \mathrm{~GB}$ RAM under MATLAB 7.0.4.

4.2. Pyramidal quantum dot. Next we consider a pyramidal quantum dot embedded in a cuboid matrix. The width of the pyramid is $12.4 \mathrm{~nm}$, and the height is $6.2 \mathrm{~nm}$. The size of the cuboid matrix is taken to be $24.8 \mathrm{~nm} \times 24.8 \mathrm{~nm} \times 18.6$ $\mathrm{nm}$. The material parameters are assumed to be same as in the last example. This is exactly the numerical example in [20].

Discretizing the Schrödinger equation with quadratic elements on a mesh of tetrahedrons, which is refined close to the boundary of the quantum dot, FEMLAB yields a rational eigenvalue problem of dimension 18990. Solving the linear eigenproblems in FAM and MFAM by eigs one obtains the upper bounds of the smallest 5 energy levels and required CPU times in Table 4.2. Notice, that the method had no problem to determine the second and third eigenvalue which is a double eigenvalue.

In the last two columns we added the eigenvalue approximations obtained by the finite volume method (FVM) in $[19,20]$ where the discrete problems have dimension 22103 and 186543, respectively. Since the FEM method yields upper bounds of the eigenvalues of the nonlinear problem (2.3) at least the eigenvalue approximations for $E_{1}, E_{2}$, and $E_{3}$ obtained by the finite volume method are less accurate than the ones 
HEINRICH VOSS

TABLE 4.1

Conical quantum dot

\begin{tabular}{|r|c|c|r|r|r|r|r|}
\hline global & $\ell$ & $\mathrm{k}$ & eigenvalue & \multicolumn{2}{|c|}{ FAM } & \multicolumn{2}{|c|}{ MFAM } \\
& & & & CPU & iterations & CPU & iterations \\
\hline 1 & 0 & 1 & 0.254585 & 6.76 & 10 & 2.97 & 4 \\
2 & 1 & 1 & 0.384162 & 10.56 & 12 & 3.84 & 4 \\
3 & 0 & 2 & 0.467239 & 13.91 & 13 & 4.77 & 4 \\
4 & 2 & 1 & 0.503847 & 12.83 & 14 & 4.23 & 4 \\
5 & 0 & 3 & 0.561319 & 19.28 & 14 & 6.32 & 4 \\
6 & 1 & 2 & 0.598963 & 20.30 & 14 & 6.59 & 4 \\
7 & 3 & 1 & 0.617759 & 16.05 & 14 & 4.91 & 4 \\
8 & 0 & 4 & 0.688563 & 25.29 & 12 & 8.40 & 4 \\
9 & 1 & 3 & 0.700778 & 25.99 & 11 & 9.27 & 4 \\
10 & 2 & 2 & 0.712773 & 24.51 & 10 & 9.96 & 4 \\
11 & 0 & 5 & 0.713677 & 11.86 & 5 & 7.14 & 3 \\
12 & 1 & 4 & 0.716805 & 14.23 & 6 & 9.14 & 4 \\
\hline
\end{tabular}

TABLE 4.2

Pyramidal quantum dot

\begin{tabular}{|r|r|r|r|r|r|r|r|}
\hline global & eigenvalue & \multicolumn{2}{|c|}{ FAM } & \multicolumn{2}{|c|}{ MFAM } & \multicolumn{2}{|c|}{ FVM } \\
& & CPU & iterations & CPU & iterations & 22103 & 186543 \\
\hline 1 & 0.3981 & 163.41 & 8 & 61.61 & 3 & 0.4017 & 0.3988 \\
2 & 0.5747 & 235.69 & 8 & 117.34 & 4 & 0.5767 & 0.5748 \\
3 & 0.5747 & 212.24 & 8 & 105.68 & 4 & 0.5767 & 0.5748 \\
4 & 0.6864 & 227.45 & 8 & 117.47 & 4 & 0.6842 & 0.6852 \\
5 & 0.6980 & 242.57 & 8 & 121.59 & 4 & 0.6992 & 0.6977 \\
\hline
\end{tabular}

obtained with the FEM.

5. Conclusions. We have analyzed the full approximation method for computing the energy levels and the wave functions of quantum dots assuming the effective one-band Hamiltonian approximation, the position and energy dependent quasiparticle effective mass approximation, the finite hard wall confinement potential, and the Ben Daniel-Duke boundary condition. The method converges globally and linear. It can be accelerated considerably taking advantage of the Rayleigh functional.

\section{Appendix A. Proof of Theorem 3.1}

We first prove that the Full Approximation Method converges alternatingly to the $k$ :th smallest eigenvalue $E_{k}$ of $(2.3)$ for the initial value $E^{0}=0$.

We already mentioned that for $c_{j}>0, j \in\{m, q\}$ the eigenvalue $\lambda_{k}(\mu)$ of (3.1) is positive for $\mu \geq 0$, and that the function $\mu \mapsto \lambda_{k}(\mu)$ is monotonically decreasing. Hence, $E^{1}=\lambda_{k}(0)>0, E^{2}=\lambda_{k}\left(E^{1}\right)>0=E^{0}$, and $E^{3}=\lambda_{k}\left(E^{2}\right) \leq \lambda_{k}\left(E^{0}\right)=E^{1}$, and it follows by induction that

$$
E^{0}<E^{2} \leq \cdots \leq E^{2 n-2} \leq E^{2 n} \leq E^{2 n+1} \leq E^{2 n-1} \leq \ldots E^{3} \leq E^{1}
$$

Let

$$
E_{*}:=\lim _{n \rightarrow \infty} E^{2 n} \quad \text { and } \quad E^{*}:=\lim _{n \rightarrow \infty} E^{2 n+1} .
$$


Then by the continuous dependence of $\lambda_{k}(\mu)$ on $\mu$ it follows that $E_{*}=\lambda_{k}\left(E^{*}\right)$ and $E^{*}=\lambda_{k}\left(E_{*}\right)$, and the convergence (3.5) follows if we show $E_{*}=E^{*}$.

Assume that $E^{*}>E_{*}$. Let $W$ be a $k$ dimensional subset of $H_{0}^{1}(\Omega)$ such that

$$
E_{*}=\lambda_{k}\left(E^{*}\right)=\min _{\operatorname{dim} V=k} \max _{\Phi \in V, \Phi \neq 0} \frac{a\left(\Phi, \Phi ; E^{*}\right)}{b(\Phi, \Phi)}=\max _{\Phi \in W, \Phi \neq 0} \frac{a\left(\Phi, \Phi ; E^{*}\right)}{b(\Phi, \Phi)},
$$

and let $\hat{\Phi} \in W$ such that

$$
\frac{a\left(\hat{\Phi}, \hat{\Phi}, E_{*}\right)}{b(\hat{\Phi}, \hat{\Phi})}=\max _{\Phi \in W, \Phi \neq 0} \frac{a\left(\Phi, \Phi, E_{*}\right)}{b(\Phi, \Phi)} .
$$

Then it follows

$$
\begin{aligned}
E^{*}-E_{*} & =\min _{\operatorname{dim} V=k} \max _{\Phi \in V, \Phi \neq 0} \frac{a\left(\Phi, \Phi ; E_{*}\right)}{b(\Phi, \Phi)}-\max _{\Phi \in W, \Phi \neq 0} \frac{a\left(\Phi, \Phi ; E^{*}\right)}{b(\Phi, \Phi)} \\
& \leq \max _{\Phi \in W, \Phi \neq 0} \frac{a\left(\Phi, \Phi ; E_{*}\right)}{b(\Phi, \Phi)}-\max _{\Phi \in W, \Phi \neq 0} \frac{a\left(\Phi, \Phi ; E^{*}\right)}{b(\Phi, \Phi)} \\
& \leq \frac{a\left(\hat{\Phi}, \hat{\Phi} ; E_{*}\right)-a\left(\hat{\Phi}, \hat{\Phi} ; E^{*}\right)}{b(\hat{\Phi}, \hat{\Phi})}
\end{aligned}
$$

For $j \in\{m, q\}$ we have

$$
\frac{2}{E_{*}+c_{j}}-\frac{2}{E^{*}+c_{j}}=\frac{E^{*}-E_{*}}{E_{*}+c_{j}} \cdot \frac{2}{E^{*}+c_{j}}
$$

and

$$
\frac{2}{E_{*}+c_{j}+\Delta_{j}}-\frac{2}{E^{*}+c_{j}+\Delta_{j}}=\frac{E^{*}-E_{*}}{E_{*}+c_{j}+\Delta_{j}} \cdot \frac{2}{E^{*}+c_{j}+\Delta_{j}},
$$

and therefore

$$
\frac{1}{m_{j}\left(E_{*}\right)}-\frac{1}{m_{j}\left(E^{*}\right)} \leq\left(E^{*}-E_{*}\right) \frac{1}{E_{*}+c_{j}} \cdot \frac{1}{m_{j}\left(E^{*}\right)}
$$

Hence,

$$
\begin{aligned}
& a\left(\hat{\Phi}, \hat{\Phi} ; E_{*}\right)-a\left(\hat{\Phi}, \hat{\Phi} ; E^{*}\right) \\
& =\left(\frac{\hbar^{2}}{2 m_{q}\left(E_{*}\right)}-\frac{\hbar^{2}}{2 m_{q}\left(E^{*}\right)}\right) \int_{\Omega_{q}}\|\nabla \hat{\Phi}\|_{2}^{2} d x+\left(\frac{\hbar^{2}}{2 m_{m}\left(E_{*}\right)}-\frac{\hbar^{2}}{2 m_{m}\left(E^{*}\right)}\right) \int_{\Omega_{m}}\|\nabla \hat{\Phi}\|_{2}^{2} d x \\
& \leq\left(E^{*}-E_{*}\right) \max _{j \in\{m, q\}} \frac{1}{E_{*}+c_{j}}\left(\frac{\hbar^{2}}{2 m_{q}\left(E^{*}\right)} \int_{\Omega_{q}}\|\nabla \hat{\Phi}\|_{2}^{2} d x+\frac{\hbar^{2}}{2 m_{m}\left(E^{*}\right)} \int_{\Omega_{m}}\|\nabla \hat{\Phi}\|_{2}^{2} d x\right) \\
& \leq\left(E^{*}-E_{*}\right) \max _{j \in\{m, q\}} \frac{1}{E_{*}+c_{j}} a\left(\hat{\Phi}, \hat{\Phi} ; E^{*}\right),
\end{aligned}
$$

and from (A.3) we obtain the contradiction

$$
\begin{aligned}
E^{*}-E_{*} & \leq\left(E^{*}-E_{*}\right) \max _{j \in\{m, q\}} \frac{1}{E_{*}+c_{j}} \frac{a\left(\hat{\Phi}, \hat{\Phi} ; E^{*}\right)}{b(\hat{\Phi}, \hat{\Phi})} \\
& \leq\left(E^{*}-E_{*}\right) \max _{j \in\{m, q\}} \frac{1}{E_{*}+c_{j}} E_{*}<E^{*}-E_{*} .
\end{aligned}
$$


The linear convergence follows from $\lim _{n \rightarrow \infty} E^{n}=E_{k}$ and the inequalities

$$
\begin{gathered}
E_{k}-E^{2 n} \leq\left(E^{2 n-1}-E_{k}\right) \max _{j \in\{m, q\}} \frac{E^{2 n}}{E_{k}+c_{j}} \\
E^{2 n+1}-E_{k} \leq\left(E_{k}-E^{2 n}\right) \max _{j \in\{m, q\}} \frac{E_{k}}{E^{2 n}+c_{j}}
\end{gathered}
$$

which are proved in the same way as inequality (A.5)

If $F^{0}$ is an arbitrary initial value satisfying $F^{0}<E_{k}$ then there exists $\ell \in \mathbb{N}$ such that $E^{2 \ell-2} \leq F^{0}<E^{2 \ell}$ where $E^{n}$ denotes the FAM iterates with initial value $E^{0}=0$, and the convergence of FAM follows from the monotonicity of $\mu \mapsto \lambda_{k}(\mu)$. If the initial approximation $F^{0}$ satisfies $F^{0}>E_{k}$, then $F^{1}<E_{k}$, and the statement follows by the same argument. 


\section{REFERENCES}

[1] Y. Li, O. Voskoboynikov, C.P. Lee, and S.M. Sze, Comput.Phys.Comm. 141 (2001) 66

[2] Y. Li, O. Voskoboynikov, C.P. Lee, and S.M. Sze, Solid State Commun. 120 (2001) 79

[3] Y. Li, O. Voskoboynikov, C.P. Lee, S.M. Sze, and O. Tretyak, J.Appl.Phys. 90 (2001) 6416

[4] Y. Li, J.-L. Liu, O. Voskoboynikov, C.P Lee, and S.M. Sze, Comput.Phys.Comm. 140 (2001) 399

[5] Y. Li, O. Voskoboynikov, C.P. Lee, S.M. Sze, and O. Tretyak, Jpn.J.Appl.Phys. 41 (2002) 2698

[6] Y. Li, O. Voskoboynikov, C.P. Lee, S.M. Sze, and O. Tretyak, Eur.Phys.J. B 28 (2002) 475

[7] Y. Li, O. Voskoboynikov, J.-L. Liu, C.P. Lee, and S.M. Sze, Techn. Proc. of the 2001 Internat. Conference on Modeling and Simulation of Microsystems, vol 1, (Nano Science and Technology Institute), 2001, p 562

[8] I. Filikhin, E. Deyneka, B. and Vlahovic, Model.Simul.Mater.Sci.Eng. 12 (2004) 1121

[9] Y. Li, J. Comput. Electronics 2 (2003) 49

[10] Y. Li, O. Voskoboynikov, and C.P. Lee, Techn. Proc. of the 2002 Internat. Conference on Modeling and Simulation of Microsystems, vol 1, (Nano Science and Technology Institute), 2002 , p 540

[11] O. Voskoboynikov, Y. Li, H.-M. Lu, C.-F. Shih and C.P. Lee, Phys. Review B 66 (2002) 155306

[12] E.O. Kane, J. Phys. Chem. Solids 1 (1957) 249

[13] G. Bastard, Wave Mechanics Applied to Semiconductor Heterostructures (Les editions de physique, Les Ulis Cedex 1988)

[14] S.L. Chuang, Physics of Optoelectronic Devices (John Wiley \& Sons, New York 1995)

[15] P. Harrison, Quantum Wells, Wires and Dots. Theoretical and Computational Physics (John Wiley \& Sons, Chichester 2000)

[16] K.P. Hadeler, Arch.Rat.Mech.Anal. 30 (1968) 297

[17] H. Voss H and B. Werner Math.Meth.Appl.Sci. 4 (1982) 415

[18] J.M. Ortega and W.C. Rheinbold, Iterative Solution of Nonlinear Equations in Several Variables (Academic Press, Boston 1970)

[19] T.-M. Hwang, W.-W. Lin, W.-C. Wang, and W. Wang, J. Comput.Phys. 196(2004) 208

[20] T.-M. Hwang, W.-W. Lin, W.-C. Wang, and W. Wang, in: Proceedings of the European Congress on Computational Methods in Applied Sciences and Engineering, (Jyväskylä, Finland) 2004

[21] FEMLAB 2004 Version 3.1. (Burlington: COMSOL Inc.)

[22] R.B. Lehoucq, D.C. Sorensen, and C. Yang, ARPACK Users' Guide. Solution of Large-Scale Eigenvalue Problems with Implicitly Restarted Arnoldi Methods (SIAM, Philadelphia 1998)

[23] Levinshtein M, Rumyantsev S, and Shur M ( editors), Handbook Series on Semiconductor Parameters (World Scientific, Singapore 1999) 